\title{
The Step-Wise Martensite to Austenite Reversible Transformation Stimulated by a Stress State
}

\author{
G. Airoldi***, A. Corsi**** and G. Riva**, (') \\ * Dipartimento di Fisica, Università di Milano, via Celoria 16, 20133 Milano, Italy \\ ** Istituto Nazionale per la Fisica della Materia, Unità di Milano Università, via Celoria 16, \\ 20133 Milano, Italy
}

\begin{abstract}
In shape memory alloys the Thermoelastic Martensitic Transformation (TMT), intrinsically step-wise, can be either thermally induced or stress-induced. Moreover, it has already been shown that an extrinsic fragmentation of thermally induced transformation can be stimulated ("Step-wise Martensite to Austenite Reversible Transformation", SMART) by means of Incomplete Cycles on Heating (ICHs): the SMART transformation shows "micromemory" features that are reversible.

SMART phenomenology appears nowadays quite a general property of shape memory alloys: it has been highlighted in several shape memory alloys, notably in NiTi, NiTiCu, NiTiFe, AgCd and CuAlZn. The relevance of the hysteresis cycle width has been nicely correlated with the key features of SMART.

SMART phenomenology induced by stress has not yet received great attention and stimulated the present investigation: NiTi wires were used to investigate the micromemory behavior in both thermally induced and stress induced martensitic transformations.

Evidence is provided for several stress-induced SMART key features, similar to those found in the thermally induced transformation. In stress-induced SMART, evidence is provided also for micromemory effects related to the direct transformation, with no thermal analogue.
\end{abstract}

\section{INTRODUCTION}

The unusual mechanical properties (shape memory effect, pseudoelasticity) of NiTi alloys are due to the Thermoelastic Martensitic Transformation (TMT), a first-order solid-solid diffisionless transformation which involves two different solid phases: the martensite ( $M$ phase, monoclinic), thermodynamically stable at $T<M_{f}$, and the parent phase ( $P$ phase, cubic), stable at $T>A_{f}$, where an applied stress can act as the driving force for the $P \rightarrow M$ transformation. The TMT is intrinsically step-wise [1-3]: this can be understood by considering the thermoelastic equilibrium that governs the transformation.

In recent years evidence has also been found for an extrinsic thermally induced stimulated step-wise fragmentation process [4-12]. Stimulated fragmentation consists in the possibility of inducing kinetic stops during the transformation, performing previous incomplete transformations.

The stimulated step-wise transformation, put into evidence for the first time in the thermally induced transformation of NiTi alloys [4, 5], was defined Step-wise Martensite to Austenite Reversible Transformation (SMART). SMART is a consequence of a partial reverse $\mathbf{M} \rightarrow \mathbf{P}$ transformation due to an incomplete cycle on heating ( $\mathrm{ICH}$ ), with a low temperature limit below $\mathbf{M}_{\mathrm{f}}$ and a high temperature limit $T_{I C H}$ within $A_{s}$ and $A_{f}$. The ICH procedure induces a stop in the kinetics of the successive complete $M \rightarrow P$ transformation, at a temperature somewhat higher than $\mathrm{T}_{\mathrm{ICH}}$ : SMART shows an overheating with respect to the previous and the subsequent cycles. Since the martensite records the $T_{I C H}$, this phenomenology has been given the name of "micromemory". The "micromemory" is wiped out after the SMART: the next $\mathrm{M} \rightarrow \mathrm{P}$ complete transformation, following SMART, restore the previous kinetics. If a number $\mathrm{N}$ of ICHs with different stop temperatures is performed, $\mathrm{N}$ stops are found in the SMART. There is no limitation on the number of ICHs, except the resolution detection limit: the stop temperatures of ICHs, however, must be selected in a decreasing rank [4]

(1) Present address: Pirelli Coordinamento Pneumatici, viale Sarca 222, 20126 Milano, Italy 
The SMART key features suggest that the kinetic stop is localized in the material, with a position related to the position of the transformation interfaces at $\mathrm{T}_{\mathrm{ICH}}$. Performing a sequence of ICHs, with $\mathrm{T}_{\mathrm{ICH}}$ in a decreasing rank, guarantees that the position of the transformation interface at each $T_{\text {ICH }}$ does not overlap that of the previous ICH. The presence of overheating in SMART suggests that the ICH procedure modifies the thermoelastic balance, causing a lack of driving force in the region of the kinetic stop. The thermoelastic balance which governs the transformation is restored after SMART, when the micromemory is wiped out. X-ray diffraction and electrical resistance investigations have clearly shown [8, 9] that thermally induced SMART is not related to kinetics barriers, but is chiefly related to the elastic relaxation processes introduced by the $\mathrm{ICH}$ procedure.

SMART phenomenology appears nowadays quite a general property of shape memory alloys: it has been highlighted in several shape memory alloy systems, notably in NiTiFe [7], NíTiCu [9], AgCd [7] and $\mathrm{CuAlZn}[10,11]$. The relevance of the hysteresis cycle width has been nicely correlated with the key features of SMART [12].

Stress-induced SMART phenomenology has not yet received great attention, except for the micromemory steps found in stress-induced martensite [13]. In order to discover if stress can act as a thermodynamic state variable in the fragmentation of the reverse stress-induced $M \rightarrow P$ transformation the stimulated stress-induced fragmentation has been investigated in NiTi alloys.

The hysteresis cycle of the stress induced martensite in a typical NiTi alloy in the pseudoelastic domain is shown in fig. 1a. After a linear stress-strain range typical of the parent phase, at a critical stress value, "the transformation stress", the stress induced martensitic transformation starts and proceeds at constant stress. The upper and the lower plateaux correspond to the direct $\mathbf{P} \rightarrow \mathbf{M}$ and reverse $\mathbf{M} \rightarrow \mathbf{P}$ stressinduced transformations.

In order to investigate SMART phenomenology in the stress-induced transformation, a partial recovery of the transformation is required (the procedure is called "Incomplete Cycle on Recovery", ICR) with at least one stop in the reverse $\mathrm{M} \rightarrow \mathrm{P}$ transformation. In this case the loop will have a start/finish high deformation limit where the specimen is totally in the $M$ phase and an inversion point at $\varepsilon_{\text {ICR }}$ within the

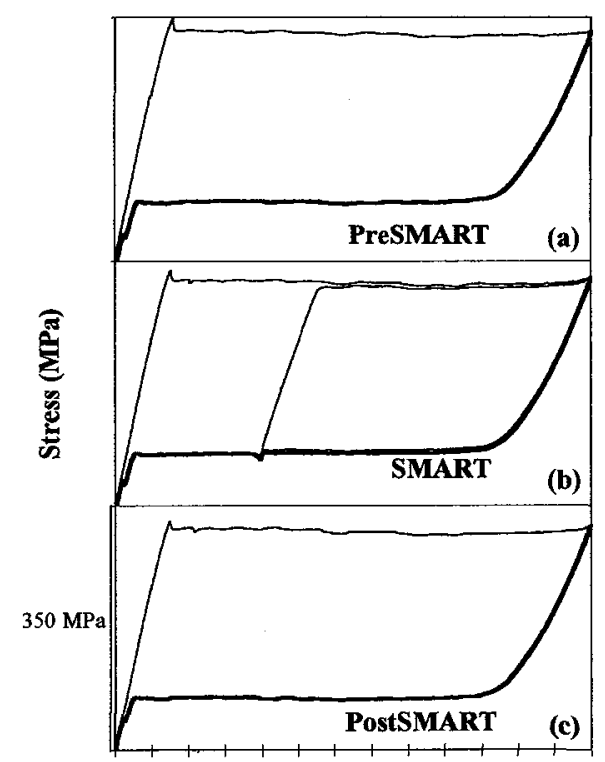

$\begin{array}{llllllllllllll}0 & 0.5 & 1 & 1.5 & 2 & 2.5 & 3 & 3.5 & 4 & 4.5 & 5 & 5.5 & 6 & 6.5\end{array}$

Strain (\%)

Figure 1 Stress-induced transformation. Procedure $\mathrm{Ib}$ : (a) mechanical behavior before the procedure; (b) during SMART and (c) after SMART. In (b) the ICR cycle is also plotted. reversion plateau (see fig. 1b). By analogy, SMART phenomenology, if it also exists in stress-induced transformations, should be found in the following $M \rightarrow P$ complete transformation on unloading.

\section{EXPERIMENTAL DETAILS}

Commercial nearly equiatomic NiTi wires $\left(0.46 \times 0.64 \mathrm{~mm}^{2}\right.$ in section) were used to investigate micromemory behavior in both thermally induced and stress induced martensitic transformations.

The thermally induced martensitic transformation was analyzed by means of Differential Scanning Calorimetry measurements using a Perkin Elmer DSC Lab 7, with a calorimetric sensitivity of $\pm 0.01 \mathrm{~mW}$. The investigated temperatures ranged from $-70^{\circ} \mathrm{C}$ to $50^{\circ} \mathrm{C}$, using a cooling system Intracooler II. The calorimeter was calibrated using an ad hoc calibration method developed for shape memory alloys [14]. A $0.083{ }^{\circ} \mathrm{C} / \mathrm{sec}$ rate was adopted.

The stress induced martensitic transformation was investigated performing tensile tests with a ZWICK 1445 testing machine (load cell $500 \mathrm{~N}$ ). Each tensile test at a constant crosshead speed $\left(1.6 \times 10^{-5} \mathrm{~m} / \mathrm{s}\right)$ was performed using a new specimen, with typical gauge length of $100 \mathrm{~mm}$. The specimen elongation was measured via the crosshead travel monitor, with resolution $1 \mu \mathrm{m}$. The test 


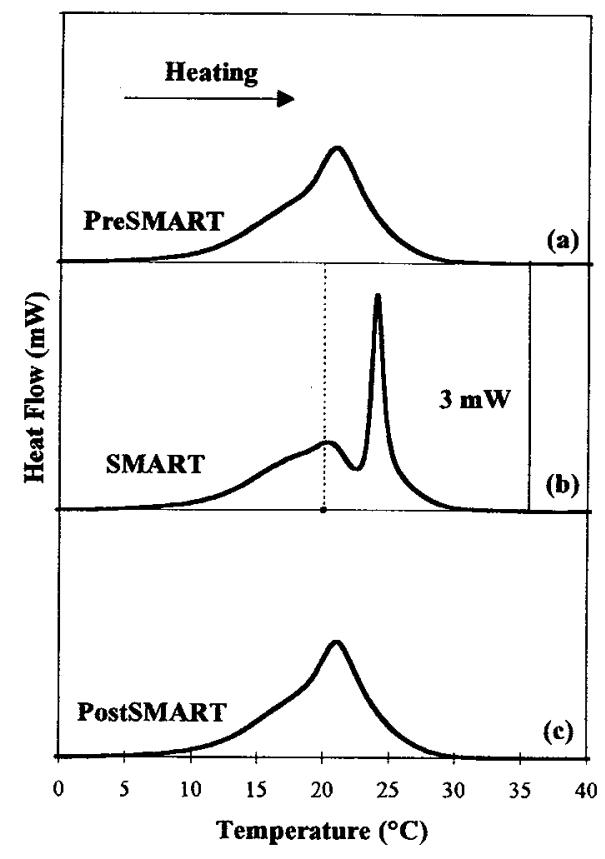

Figure 2 Thermally induced transformation. Typical DSC scans following procedure Ia: (a) before the procedure; (b) during SMART and (c) after SMART. The full dot indicates the stop temperature selected to perform the ICH.

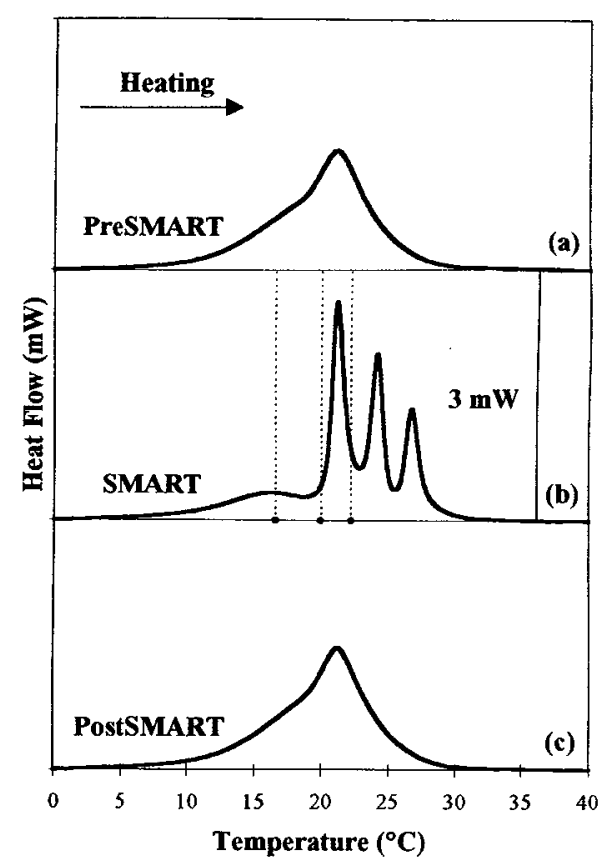

Figure 3 Thermally induced transformation. Typical DSC scans following procedure IIa: (a) before the procedure; (b) during SMART and (c) after SMART. Full dots indicate the stop temperatures selected to perform the ICHs.

temperature was held constant at $40 \pm 0.1^{\circ} \mathrm{C}$ using a circulating liquid thermostatic chamber equipped with a Lauda RC20 thermostat.

\section{PROCEDURES}

A typical DSC thermogram on heating of the investigated alloy is shown in fig. $2 \mathrm{a}\left(\mathrm{A}_{\mathrm{s}}=13.4^{\circ} \mathrm{C}\right.$, $A_{f}=25.5^{\circ} \mathrm{C}$ ). The temperature chosen for tensile tests, $T=40^{\circ} \mathrm{C}$, is somewhat higher than the $M \rightarrow P$ finish temperature (fig. 2a) in order to guarantee the presence of the stress-induced martensite and good transformation pseudoelasticity.

In both thermal and stress-induced transformations, micromemory properties have been investigated following three different procedures.

In thermally induced transformations:

Ia. a single ICH with a stop temperature corresponding to $50 \%$ of the transformed martensite (evaluated in the previous complete transformation before ICH) (see fig. 2);

IIa. three ICH's with different stop temperatures, chosen in decreasing rank, which correspond respectively to 75,50 and $25 \%$ of the transformed martensite (see fig. 3);

IIIa. six ICH's with the same stop temperature, which corresponds to $50 \%$ of transformed martensite (this procedure, with all the ICH's characterized by the same stop temperature has been defined HAMMER) (see fig. 4).

In stress induced transformations:

Ib. a single ICR with a stop deformation corresponding to $60 \%$ of the transformed martensite (evaluated in the previous complete transformation before ICR) (see fig. 1);

IIb. three ICR's with different stop deformations, chosen in increasing rank, which correspond respectively to 60,40 and $20 \%$ of the transformed martensite (see fig. 5); 


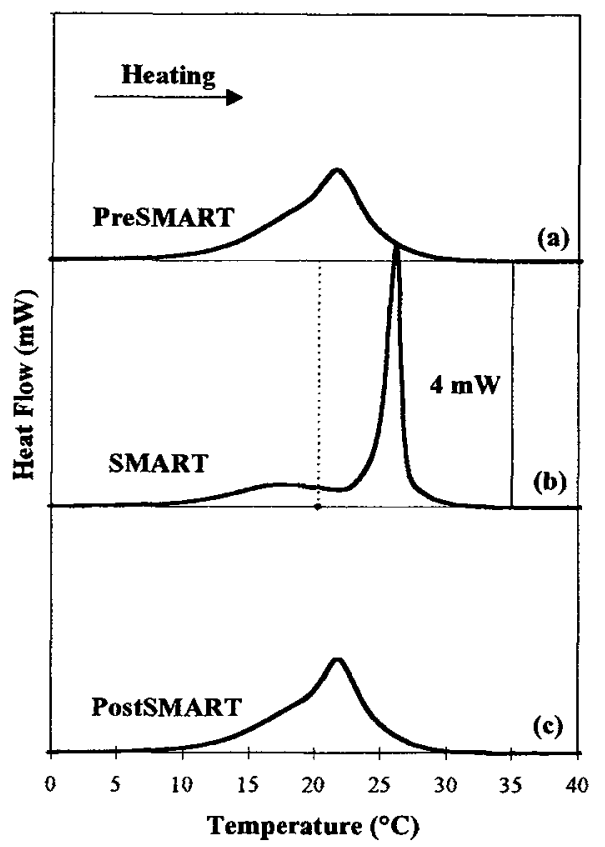

Figure 4 Thermally induced transformation. Typical DSC scans following procedure IIIa: (a) before the procedure; (b) during SMART, after the HAMMER procedure with 6 cycles, and (c) after SMART. The full dot indicates the stop temperature selected to perform the ICH.

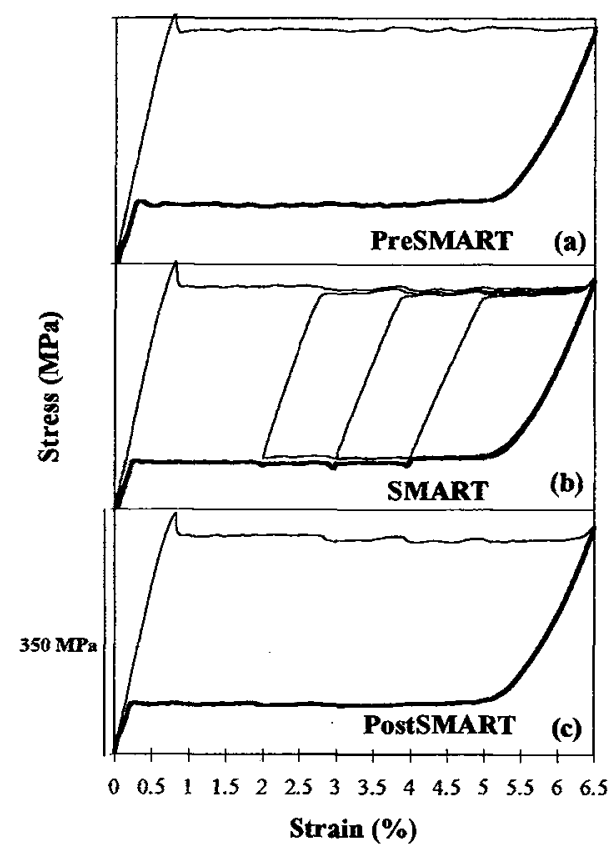

Figure 5 Stress induced transformation. Typical mechanical behavior following procedure Ilb: (a) before the procedure; (b) during SMART and (c) after SMART. In (b) the ICR cycles are also plotted.

IIIb. ten ICR's with the same stop deformation which corresponds to $60 \%$ of transformed martensite (this procedure, with all the ICR's characterized by the same stop deformation has been defined HAMMER) (see fig. 6).

\section{RESULTS AND DISCUSSION}

The results obtained here on thermally induced SMART (figs. 2, 3, 4) confirm previous investigations performed on several different shape memory alloys $[9,11,12]$. The micromemory is the consequence of a kinetic stop in the reverse transformation, is associated with the reverse transformation from the martensite to parent phase, and appears as a local property. The minima of the DSC scans are strictly related to the stop temperatures of the ICH's, even if with overheating: the temperatures of the minima of the SMART scans are somewhat higher than the stop temperatures of the ICH's (indicated by full dots in the figures). If $\mathrm{N}$ ICH's with different stop temperatures are performed, $\mathrm{N}$ minima are found in the SMART thermogram (fig. 3b). All $\mathrm{N}$ minima show almost the same overheating with respect to the corresponding stop temperatures.

Stimulated fragmentation is, moreover, a reversible process: the transformation kinetics of the PreSMART (figs. 2a, 3a, 4a) is almost completely recovered in the complete cycle (figs. 2c, 3c, 4c) that follows the SMART: the overcoming of the stop temperature of an ICH wipes out the micromemory related to that $\mathrm{ICH}$.

The SMART behavior related to HAMMER procedure (procedure IIIa) is similar to that related to a single ICH procedure, but the overheating is more sizable with respect to the single ICH case (figs. $2 \mathrm{~b}$ and 4b): the HAMMER procedure induces a lack of driving force larger than that produced by the single ICH procedure.

Similar features appear in the micromemory related to the stress induced transformation. In figures $1 \mathrm{~b}$, 


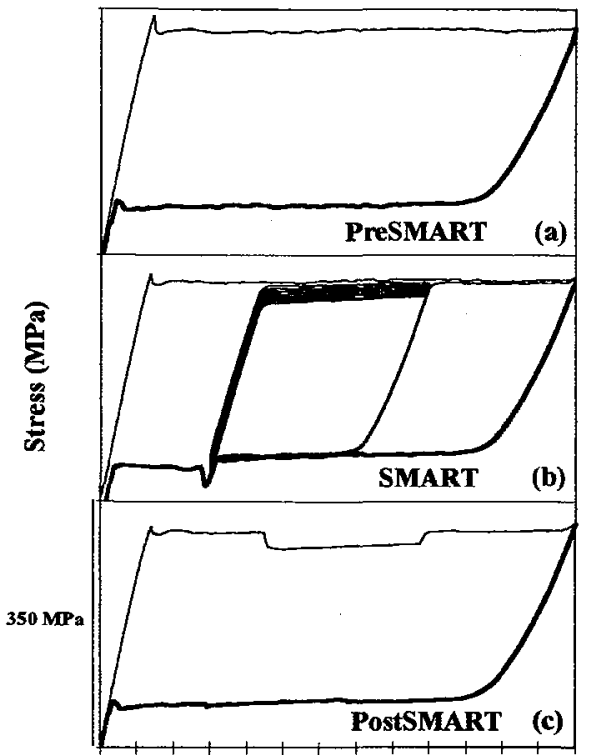

$\begin{array}{lllllllllllllll}0 & 0.5 & 1 & 1.5 & 2 & 2.5 & 3 & 3.5 & 4 & 4.5 & 5 & 5.5 & 6 & 6.5\end{array}$

Strain (\%)

Figure 6 Stress induced transformation. Typical mechanical behavior following procedure IIIb: (a) before the procedure; (b) during SMART, after the HAMMER procechure with 10 cycles, and (c) after SMART. reversible process also in stress-induced transformations: the reverse transformation kinetics in the complete cycle that precedes the ICR procedure (fig. $1 \mathrm{a}, 5 \mathrm{a}, 6 \mathrm{a}$ ) is almost completely recovered in the complete cycle (fig. 1c, 5c, 6c) that follows the SMART with the step-wise behavior. Here also, there is an apparent arbitrariness in the choice of the stop deformations. The reversibility of the process requires an increasing rank for the stop deformations, in procedures involving ICRs with different stop deformations: in figure $5 \mathrm{c}$ it may be remarked that the PostSMART curve exhibits no step-wise behavior; the overcoming of a stop deformation of an ICR wipes out the micromemory related to that ICR. Should the stop deformations be chosen in a decreasing rank, the SMART transformation would show the micromemory related just to the last ICR. This is in full analogy with the key features of the thermally induced SMART.

Similarly to thermally induced transformations, a HAMMER procedure (procedure IIIb) strengthens the results obtained performing one single ICR: the understress step (see fig. 6b) is greatly enhanced with respect to the single ICR case (fig. 1b). The HAMMER procedure appears to induce a lack of driving force larger than that produced by a single ICR stop.

In contrast to thermally induced transformations, where no evidence of micromemory associated with the direct transformation from parent to martensite phase was found [6,7], in stress-induced transformations evidence of a micromemory associated with the direct transformation is recorded. A careful examination of fig. $6 \mathrm{c}$ shows the presence of a "micromemory" in the direct transformation: the value of the transformation stress in the central region of the plateau, the region that has undergone the partial transformations, is lowered.

Moreover, the micromemory effect in the stress-induced direct transformation cannot be erased by performing complete mechanical cycles, but can be erased by் performing appropriate thermal treatment.

These aspects of the mechanical micromemory, most probably related to the creation and the erasure of preferential nucleation sites introduced with dislocations, are still an open subject of investigation.

As for as the amount of the lack of the driving force in the two transformations is concerned, from fig. 
$2 \mathrm{~b}$ it can be seen that in the single stop procedure there is $\sim 2.2^{\circ} \mathrm{C}$ overheating. From the ClausiusClapeyron relation, the stress to induce martensite modifies with temperature with a rate of $\sim 8 \mathrm{MPa} /{ }^{\circ} \mathrm{C}$, as found for these NiTi alloys [17]. So, we should expect a $\sim 16 \mathrm{MPa}$ understressing: from these investigations we obtain $12 \mathrm{MPa}$, of the same order of the expected value.

\section{CONCLUSIONS}

Step-wise stimulated fragmentation of martensitic transformations, either thermally induced or stressinduced, shows similar key features:

- the micromemory is related to the reverse transformation;

- the micromemory of partial cycles shows local character;

- the effect is erasable by performing a complete cycle following either ICHs or ICRs;

- there is no apparent limitation to the number of ICH's or ICR's that can be performed but a rank order is required for the temperature or deformation stops;

- a one-to-one correspondence exists between the ICH or ICR stops and the kinetic stops during SMART;

- in both kinds of transformations a lack of driving force appears related to partial cycles: in thermally induced transformation the evidence of this lack is given by overheating, whilst in stress-induced transformation by understressing. The amounts of the lack of driving force are in agreement.

In the stress-induced case evidence is also found for the existence of micromemory effects related to the direct transformation: this kind of micromemory has no thermal analogue and is erasable on condition that an appropriate thermal treatment is performed.

\section{Ackmowledgments}

Research was supported by Istituto Nazionale per la Fisica della Materia (I.N.F.M).

\section{References}

[1] G. Airoldi, G. Riva, ISIJ International, 29, 5 (1989) 412-416.

[2] E. Hornbogen, Z. Metallkde., 78 (1987) 352-354.

[3] R. Gotthardt, J. Stoiber, in The Martensitic Transformation in Science and Technology (edited by E. Hornbogen and N. Jost), DGM, Oberursel (1989) pp. 27-38.

[4] G. Airoldi, G. Riva, in The Martensitic Transformation in Science and Technology (edited by E. Hornbogen and N. Jost), DGM, Oberursel (1989) pp. 305-312.

[5] W.A. Johnson, J.A. Domingue, S.H. Reichman, J. Physique, C4 Coll., Suppl. No. 12 (1982) 285-290.

[6] G. Airoldi, G. Riva, Key Engineering Materials, 48 (1990) 5-16.

[7] G. Airoldi, S. Besseghini, G.Riva, "Step-wise transformations in shape memory alloys", Int. Conf. on Martensitic Transf. (ICOMAT-92), Monterey 20-24 July 1992, C.M. Wayman and J. Perkins Eds. (Monterey Institute of Advanced Studies, Carmel..1993) pp. 959-964.

[8] G. Airoldi, G. Carcano, G.Riva, J. Physique, Coll. C4, 1 (1991) 277-282.

[9] G. Airoldi, S. Besseghini, G.Riva, J. Physique, Coll. C2, 5 (1995) 483-488.

[10] A. Amengual, Scripta Metall. Mater., 26 (1992) 1795-1798.

[11] G. Airoldi, S. Besseghini, G.Riva, J. Physique, C8 Coll., 5 (1995) 877-882.

[12] G. Airoldi, S. Besseghini, G.Riva, Il Nuovo Cimento, 15D, No. 2-3 (1993) 365-374.

[13] S. Eucken, T. W. Duerig, Acta. metall. mater., 37, 8 (1989) 2245-2252.

[14] G. Airoldi, G. Riva, M. Vanelli, Journal of Thermal Analysis, 42 (1994) 781-791.

[15] G. Riva, G. Airoldi, S. Besseghini, Meccanica, 30, (1995) 495-503.

[16] J.L. Proft, K.N. Melton, T.W. Duerig, "Yield drop and snap action in a warm worked Ni-Ti-Fe alloy", Int. Conf. on Martensitic Transf. (ICOMAT-86), Nara 26-30 August 1986, I. Tamura Ed. (The Japan Institute of Metals, Aoba Aramaki, 1987) pp. 742-747.

[17] G. Airoldi, G.Riva, M. Vanelli, J. Physique, C8 Coll., 5 (1995) 1205-1210. 